\title{
ESTUDO POMOLÓGICO COMPARATIVO DAS CULTIVARES DE CUCURBITA*
}

\author{
LUIZ ANTONio RocheLLE **
}

\begin{abstract}
RESUMO
O presente trabalho teve por finalidade descrever comparativamente as características morfológicas dos frutos e das sementes de 13 cultivares de Cucurbita.

Para identificar e classificar botanicamente as espécies das cultivares em estudo, servimo-nos da chave descrita por Bailey, que considera entre outras, as características dos pedúnculos dos frutos e as características das sementes. Desta maneira separamos as cultivarcs em três espécies: Cucurbita moschata Duchesne com as seguintes cultivares: 'Menina-verde', 'Paca', 'Tatui', Meninaamarela', 'Canhão', 'Redonda-de-amparo' e 'Menina-creme'. Da espécie Cucurbita maxima Duchesne estudamos os frutos $e$ as sementes das cultivares 'Exposição', 'Coroa', 'Ovo-de-ganso' e 'Mogango-verde' e para Cucurbita pepo Linneu as cultivares 'Small-sugar' e 'Caserta'.

Realizamos o referido trabalho no Campo Experimental do Departamento de Agricultura-Horticultura da ESALQ, em Piracicaba, e as cultivares estudadas são as mais recomendadas pela Secção de Olericultura do Instituto Agronômico de Campinas.

Foram semeadas de cada cultivar 3 a 5 sementes por cova e repetidas por 10 vezes. De cada planta adulta colhemos 3 frutos e nos frutos anotamos as seguintes características morfológicas: coloração do epicarpo, forma, resistência da casca, dimensões e peso; semente: dimensões, número de sementes normais e anormais, peso das sementes.
\end{abstract}

\section{INTRODUÇÃO}

A família Cucurbitaceae, que compreende entre outros, os gêneros Citrullus (melancia), Cucumis (melão e pepino), Sechium (chuchú), possui também o gênero Cucurbita com aproximadamente 25 espécies, sendo destas, três realmente de valor econômico, devido as suas qualidades e o interesse que despertam no estudioso do assunto.

- Entregue para publicação em 13/7/1976.

- Departamento de Botânica - ESALg. 
De fato, os frutos de Cucurbita moschata Duchesne, onde estão todas as aboboreiras rasteiras, Cucurbita maxima Duchesne, compreendendo as morangueiras e mogangueiras, assim como Cucurbita pepo Linneu, com uma aboboreira e uma morangueira, entram em quantidade na alimentação do nosso povo, sob diversas formas. Isso, aliás, justifica nos grandes mercados brasileiros, mormente nos localizados nos Estados de São Paulo e Rio de Janeiro, o interesse pelo cultivo destas plantas e da produção de seus saborosos e nutritivos frutos.

Hoje em dia, a importância é ainda aumentada, principalmente pelo crescimento da industrialização, através da desidratação de produtos para a fabricação de sopas e para o congelamento.

Atualmente, existem determinadas exigências pelos consumidores de abóboras maduras ou secas, em relação a coloração do epicarpo, formato e peso dos frutos, os quais precisam ser uniformes e definidos, assim como a polpa deve ser enxuta, não possuir fibras e ter uma coloração acentuada.

Frutos de polpa com coloração pálida, não alcançam bons preços, nem sendo às vezes vendidos.

O presente trabalho tem por finalidade, descrever as características morfológicas dos frutos, assim como também as características morfológicas das sementes dos mesmos.

\section{MATERIAIS E METODOS}

O referido trabalho foi conduzido nas dependências do Campo Experimental do Departamento de Agricultura-Horticultura da ESALQ, em Piracicaba.

O estudo pomológico foi feito para as seguintes cultivares: Cucurbita moschata Duchesne - aboboreiras rasteiras - 'Menina-verde', 'Paca', 'Tatui', 'Menina-amarela', 'Canhão', 'Redonda-de-amparo' e 'Meninacreme'.

Cucurbita maxima Duchesne - morangueiras (frutos redondos-achatados) - 'Exposição' e 'Coroa', mogangueiras (frutos oval-alongadcs) 'Ovo-de-ganso' e 'Mogango-verde'.

Cucurbita pepo Linneu - morangueira 'Small-sugar' e aboboreira 'Caserta'.

O espaçamento foi de 3 metros nas linhas por 3 metros nas entrelinhas. As covas possuíam as seguintes dimensões: 40x40x20 centíme- 
tros e receberam 3 quilos de esterco de curral. Foram semeadas de 3 a 5 sementes (provenientes de polinização controlada) por cova. Após o desbaste, foram aplicados 30 gramas de Salitre do Chile, em cobertura e por cova.

Para a obtenção das medidas dos frutos e das sementes, utilizamo-nos de um paquímetro e de uma régua aferida; para a pesagem dos mesmos usamos uma balança Triple Beam. Para avaliarmos o grau de resistência da casca dos pepônios estudados, utilizamo-nos de um pressômetro do tipo $10 \mathrm{~B}$, da D. Ballaufmfg. Co. Inc. Washington-USA, sendo o diâmetro da ponta perfuradora igual a $2 \mathrm{~mm}$. Foram feitas 9 perfurações em cada fruto obtido, sendo 3 perfurações na região basal, 3 na região mediana e 3 na região apical.

De cada planta adulta obtivemos 3 frutos, nos quais anotamos as seguintes características:

coloração do epicarpo

forma dos pepônios

dimensões e pesos dos pepônios

resistência da casca

número de sementes normais e anormais por pepônio

dimensões e peso das sementes.

\section{RESULTADOS}

Resultados obtidos da média de 30 frutos (por cultivar) aproximadamente. Ver figura 1.

\section{Cultivares de Cucurbita moschata Duchesne.}

'Menina-verde' - Fruto com epicarpo de coloração castanha-escura, com listas verde-escuras, forma cilíndrica, recurvada, tendo uma dilatação terminal (bojo), medindo $63 \mathrm{~cm}$ de comprimento, o pescoço (porção recurvada) com $11 \mathrm{~cm}$ de diâmetro e o bojo com $18 \mathrm{~cm}$ de diâmetro; casca com resistência igual a 6 libras de pressão, pesando $6.540 \mathrm{~g}$; a polpa com espessura de $2,8 \mathrm{~cm}$, com 508 sementes normais e 42 anormais, medindo $1,5 \mathrm{~cm}$ de comprimento por $0,8 \mathrm{~cm}$ de largura, pesando $0,20 \mathrm{~g}$, com uma costela ou relevo linear presente numa das faces (longitudinais).

'Paca' - Fruto com epicarpo de coloração castanha e listras verde-escuras, forma elipsóide com o eixo maior no prolongamento do seu pedúnculo, medindo $29 \mathrm{~cm}$ de comprimento por $18 \mathrm{~cm}$ de diâmetro; casca com resistência igual a 7 libras de pressão; pesando 4.570 g; a polpa com a espessura de $5,6 \mathrm{~cm}$ na região próxima a inserção do pedúnculo, 


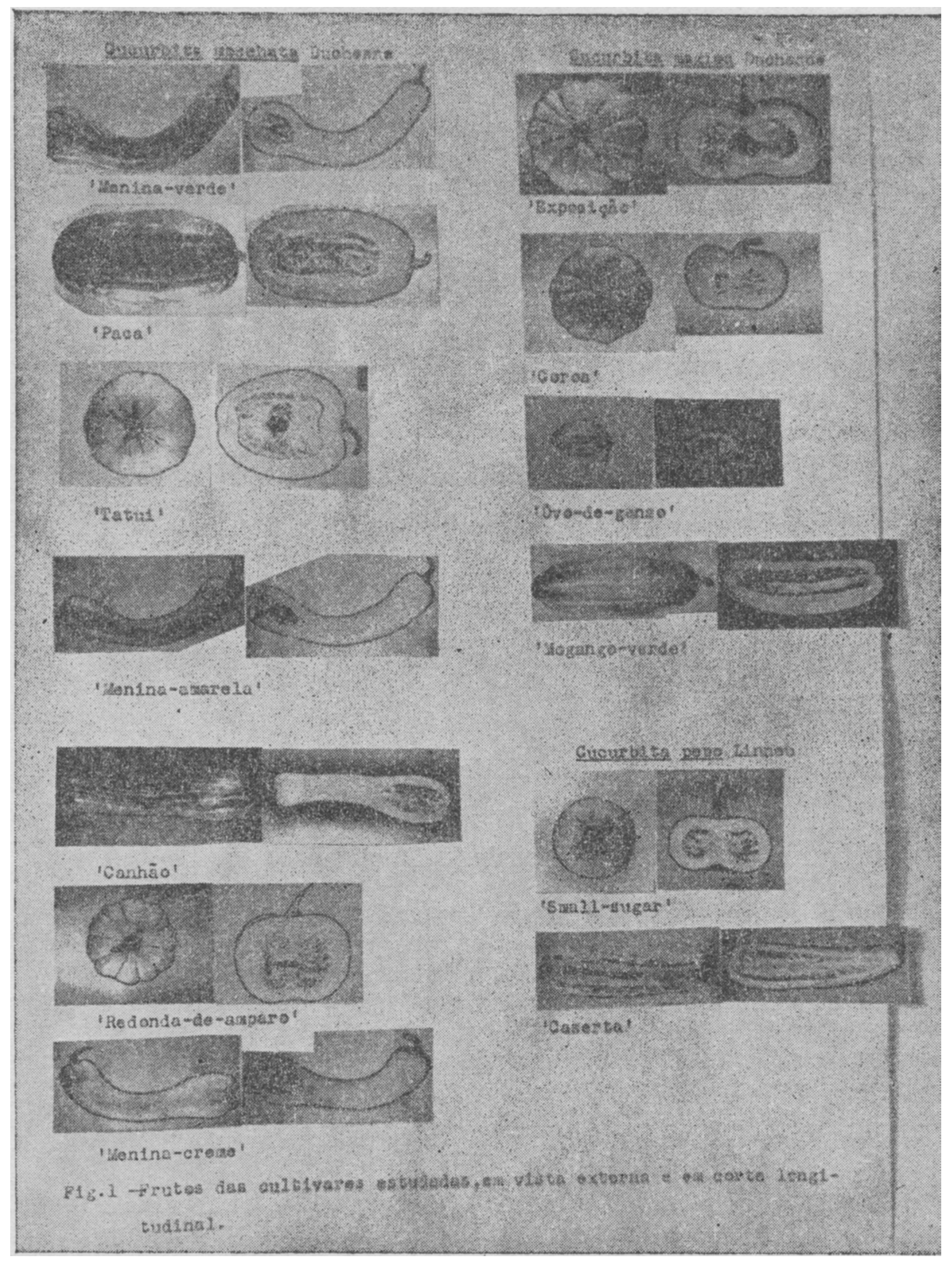


$3,6 \mathrm{~cm}$ na região mediana e $2,5 \mathrm{~cm}$ na porção próxima a inserção dos verticilos florais; com 612 sementes normais e 97 anormais, medindo $1,5 \mathrm{~cm}$ de comprimento por $0,9 \mathrm{~cm}$ de largura, pesando $0,26 \mathrm{~g}$, com uma costela ou relevo linear presente numa das faces (longitudinais).

'Tatui' - Fruto com epicarpo de coloração amarela pronunciada (às vezes clareando pouco) e com listas longitudinais, forma ovóide, medindo $20 \mathrm{~cm}$ de comprimento por $19 \mathrm{~cm}$ de largura; casca com resistência igual a 5 libras de pressão; pesando $3.620 \mathrm{~g}$; polpa com espessura de $3,8 \mathrm{~cm}$ na região próxima à inserção do pedúnculo, $2,9 \mathrm{~cm}$ na região meãiana e $2,5 \mathrm{~cm}$ na região próxima a inserção dos verticilos florais; com 428 sementes normais e 48 anormais, medindo $1,4 \mathrm{~cm}$ de comprimento por $0,7 \mathrm{~cm}$ de largura, pesando $0,16 \mathrm{~g}$.

'Menina-amarela' - Fruto com epicarpo de coloração amarela, c'e forma cilíndrica recurvada, com uma dilatação terminal (bojo), medindo $60 \mathrm{~cm}$ de comprimento, tendo o pescoço $8,0 \mathrm{~cm}$ de diâmetro e o bojo $15 \mathrm{~cm}$ de diâmetro; casca com resistência igual a 6,0 libras de pressão; pesando $4.160 \mathrm{~g}$, a polpa com espessura de $2,0 \mathrm{~cm}$ ao redor da cavidade do fruto; com 324 sementes normais e 43 anormais, medindo $1,4 \mathrm{~cm}$ de comprimento por $0,6 \mathrm{~cm}$ de largura, pesando $0,16 \mathrm{~g}$, com uma costela ou relevo linear presente numa das faces (longitudinais).

'Canhão' - Fruto com epicarpo de coloração castanha, variegado de verde-escuro, forma cilíndrica, dilatando-se na outra extremidade, medindo $71 \mathrm{~cm}$ de comprimento, com $12 \mathrm{~cm}$ de diâmetro no pescoço e $19 \mathrm{~cm}$ no bojo; casca com resistência igual a 6 libras de pressão; pesando $9.980 \mathrm{~g}$; a polpa com 3,0 cm de espessura ao redor da cavidade do fruto; com 347 sementes normais e 59 anormais, medindo $1,4 \mathrm{~cm}$ de comprimento por $0,8 \mathrm{~cm}$ de largura, pesando $0,18 \mathrm{~g}$, tendo uma costela ou relevo linear presente numa das faces (longitudinais).

'Redonda-de-amparo' - Fruto com epicarpo de coloração amarela intensa, de forma globosa, possuindo duas concavidades bem rasas, uma delas na inserção do pedúnculo e outra na região que lhe é oposta, tendo ainda gomos poucos notáveis, medindo 16 centímetros de comprimento por $17 \mathrm{~cm}$ de diâmetro; casca com uma resistência igual a 5,5 libras de pressão; pesando $2.160 \mathrm{~g}$; polpa com $2,7 \mathrm{~cm}$ de espessura na região próxima a inserção de pedúnculo, $2,6 \mathrm{~cm}$ de espessura na região mediana e $1,8 \mathrm{~cm}$ na porção próxima a inserção dos verticilos florais; com 319 sementes normais e 79 anormais, medindo $1,7 \mathrm{~cm}$ de comprimento por $0,9 \mathrm{~cm}$ de largura, pesando $0,32 \mathrm{~g}$, sendo a costela ou relevo linear muito raro nesta cultivar.

'Menina-creme' - Fruto com epicarpo de coloração creme- de forma cilíndrica recurvada, com uma dilatação terminal (bojo), medindo $59 \mathrm{~cm}$ de comprimento, tendo o pescoço $8,0 \mathrm{~cm}$ de diâmetro e o bojo $13 \mathrm{~cm}$ de diâmetro; casca com resistência igual a 6,5 libras de pressão; com um peso de $4.140 \mathrm{~g}$; a polpa com $1,8 \mathrm{~cm}$ ao redor da 
cavidade do fruto; com 330 sementes normais e 40 anormais, medindo $1,0 \mathrm{~cm}$ de comprimento por $0,6 \mathrm{~cm}$ de alrgura, com peso de $0,14 \mathrm{~g}$, com a costela ou relevo linear quase sempre ausente.

\section{Cucurbita maxima Duchesne}

'Exposição' - Fruto com epicarpo de coloração amarela-avermelhada brilhante, de forma globosa, tendo gomos longitudinais notáveis, um tanto achatada ainda, com duas concavidades uma mais profunda onde se insere o pedúnculo, a outra mais rasa na face que lhe é oposta, medindo $12 \mathrm{~cm}$ de comprimento por $23 \mathrm{~cm}$ de diâmetro; casca com 5 libras de resistência; com u mpeso de $3.820 \mathrm{~g}$; a polpa com 2,0 cm de espessura na região próxima a inserção do pedúnculo, $3,2 \mathrm{~cm}$, de espessura na região mediana e $2,2 \mathrm{~cm}$ de espessura na porção próxima à inserção dos verticilos florais; com 390 sementes normais e 75 anormais, medindo $1,5 \mathrm{~cm}$ de comprimento por $0,9 \mathrm{~cm}$ de largura, pesando $0,36 \mathrm{~g}$.

'Coroa' - Fruto com epicarpo de coloração verde-acinzentada hrilhante, de forma globosa, um tanto achatada e possuindo gomos longitudinais pouco notáveis, apresentando duas concavidades, sendo uma mais profunda onde se insere o pedúnculo, a outra mais rasa na face que lhe é oposta, medindo $12 \mathrm{~cm}$ de comprimento por $19 \mathrm{~cm}$ de diâmetro; com a casca de 6,5 libras de pressão de resistência; pesando $2.750 \mathrm{~g}$, com $2,2 \mathrm{~cm}$ de espessura na região próxima a inserção do pedúnculo, $2,8 \mathrm{~cm}$ na região mediana e $2,5 \mathrm{~cm}$ na região $\mathrm{p}$ róxima à inserção dos verticilos florais; com 345 sementes normais e 79 anormais, medindo $1,6 \mathrm{~cm}$ de comprimento por $1,0 \mathrm{~cm}$ de largura, pesando 0,42 $\mathrm{g}$.

'Ovo-de-ganso' - Fruto com epicarpo de coloração cinzenta brilhante, de forma ovóide, com gomos longitudinais não notáveis, medindo $11 \mathrm{~cm}$ de comprimento por $7,0 \mathrm{~cm}$ de diâmetro; casca com resistência superior a 10 libras de pressão; com um peso de $256 \mathrm{~g}$; a polpa com $1,2 \mathrm{~cm}$ de espessura na região próximaainserção do pedúnculo; com $1,2 \mathrm{~cm}$ de espessura na região mediana e $1,5 \mathrm{~cm}$ na região próxima a inserção dos verticilos florais; com 59 sementes normais e 13 anormais, medindo $1,4 \mathrm{~cm}$ de comprimento por $1,0 \mathrm{~cm}$ de largura e pesando $0,28 \mathrm{~g}$.

'Mogango-verde' - Fruto com epicarpo de coloração verde-acinzentada brilhante com listras longitudinais não muito visíveis; de forma elipsóide alongada, quase cilíndrica, com gomos longitudinais não notáveis, medindo $35 \mathrm{~cm}$ de comprimento por $11 \mathrm{~cm}$ de diâmetro; casca com resistência igual a 7 libras de pressão, com um peso de $2.420 \mathrm{~g}$; a polpa com $2,4 \mathrm{~cm}$ de espessura na região próxima a inserção do pedúnculo, $2,4 \mathrm{~cm}$ na região mediana e $3,4 \mathrm{~cm}$ na porção próxima a inserção dos verticilos florais; com 233 sementes normais e 86 anormais, medindo $1,5 \mathrm{~cm}$ de comprimento por $1,0 \mathrm{~cm}$ de largura e pesando $0,34 \mathrm{~g}$. 


\section{Cucurbita pepo Linneu}

'Small-sugar' - Fruto com epicarpo de coloração amarelo-alaranjada brilhante, de forma globosa bem achatada, com duas concavidades pouco profundas, uma onde se insere o pedúnculo e a outra na face que lhe é oposta; com $9,0 \mathrm{~cm}$ de comprimento por $15 \mathrm{~cm}$ de diâmetro; casca com resistência igual a 7,5 libras de pressão, com um peso de $1.132 \mathrm{~g}$; polpa com 1,8 cm de espessura na região próxima a inserção do pedúnculo, $1,9 \mathrm{~cm}$ na região mediana e $1,5 \mathrm{~cm}$ na região próxima a inserção dos verticilos florais; com 297 sementes normais e 49 anormais, medindo $1,6 \mathrm{~cm}$ de comprimento por $0,9 \mathrm{~cm}$ de largura e com $0,22 \mathrm{~g}$ de peso.

'Caserta' - Fruto com epicarpo de coloração amarelo-clara com manchas verdes, de forma claviforme, medindo $32 \mathrm{~cm}$ de comprimento por $9,0 \mathrm{~cm}$ de diâmetro; casca com resistência igual a 6 libras de pressão, com $1.320 \mathrm{~g}$ de peso; polpa com $3,0 \mathrm{~cm}$ de espessura na região próxima a inserção do pedúnculo, $1,9 \mathrm{~cm}$ na região mediana e $2,1 \mathrm{~cm}$ na região próxima a inserção dos verticilos florais; 115 sementes normais e 54 anormais, medindo $1,6 \mathrm{~cm}$ de comprimento por $0,9 \mathrm{~cm}$ de largura e pesando $0,22 \mathrm{~g}$.

\section{CONCLUSÕES}

As seguintes conclusões são tiradas:

1. A caracterização morfológica das cultivares estudadas, pode ser realizada pelo estudo dos frutos das mesmas.

2. As sementes das cultivares estudadas, oferecem excelente subsídio à caracterização morfológica.

3. As cultivares que apresentaram maiores dimensões foram: 'Canhão', 'Menina-verde', 'Menina-amarela' e 'Menina-creme', todas pertencentes a Cucurbita moschata Duchesne, 'Mogango-verde' da espécie Cucurbita maxima Duchesne e "Caserta" da espécie Cucurbita pepo Linneu.

4. As cultivares que apresentaram menores dimensões foram: 'Redonda-de-amparo', da espécie Cucurbita moschata Duchesne, 'Ovo-de-ganso' pertencente a Cucurbita maxima Duchesne e 'Small-sugar' pertencente a Cucurbita pepo Linneu.

5. Em peso as maiores cultivares foram:

'Canhão' (Cucurbita moschata Duchesne), 'Exposição' (Cucurbita maxima Duchesne), 'Caserta' e 'Small-sugar' (Cucurbita pepo Linneu). 
6. As cultivares que apresentaram menores pesos foram:

'Redonda-de-amparo' (Cucurbita moschata Duchesne) e 'Ovo-de-ganso' (Cucurbita máxima Duchesne).

7. A cultivar 'Ovo-de-ganso' (Cucurbita maxima Duchesne) apresentou a casca mais resistente, vindo a seguir 'Small-sugar' (Cucurbita pepo Linneu) e 'Paca' (Cucurbita moschata Duchesne) .

8. O maior número de sementes normais foi encontrado nas cultivares 'Paca' (Cucurbita moschata Duchesne), 'Exposição' (Cucurbita maxima Duchesne) e 'Small-sugar' (Cucurbita pepo Linneu).

9. A cultivar que apresentou o menor número de sementes normais foi a 'Ovo-de-ganso' (Cucurbita maxima Duchesne).

10. As cultivares 'Coroa', 'Exposição', 'Mogango-verde', todas pertencentes a Cucurbita maxima Duchesne, apresentaram as sementes mais pesadas.

11. Todas as cultivares consideradas, apresentaram os frutos com a casca firme e a polpa enxuta.

\section{SUMMARY}

COMPARATIVE POMOLOGIC STUIYY OF CUCURBITA CULTIVARS

This paper deals with characterization of fruits of 13 cultivars belonging to 3 species: Cucurbita moschata Duchesne, Cucurbita maxima Duchesue and Cucurbita pepo Linneu. It was used seeds from controlled pollination. It was considered following characteristics of fruits: epicarp, shape, weight, resistance of the bark, dimensions and thickness of pulp; For seerls were considered: number, dimensions and weight.

\section{LITERATURA CITADA}

BAILEY, L.H. 1964 -- Manual of cultivated plants. New York, The Macmillan Company. $1116 \mathrm{p}$.

CASTETTER, E.F.; ERWIN, A.T. 1927 - A systematic study of squashes and pumpkins. Iowa, p. 107-135.

DUPAIGNE, P. 1961 - Sur la détermination de la fermeté des fruits aumoyen du penetromètre. Fruits. 16, 3: 107-111.

ENcileR, A. $196+$ - Syllabus der Pflanzenfamilien. Berlin, 2: 341-345. 
GOURLEY, J.H.; HOWLETT, F.S. 1941 - A classification of fruits. New York, The Macmillan Company.

QUER, P.F. 1970 - Dicionário de Botânica. Rio de Janeiro, Editorial Labor SA. 1244 p.

ROCHELLE, L.A. 1973 - Descrição taxonômica de cultivares de Cucurbita moschata Duchesne. Anais da E.S.A. "Luiz de Queiroz", 30: 129-161.

ROCHELLE, L.A. 1974 - Descrição taxonômica de cultivares de Cucurbita maxima Duchesne e Cucurbita pepo Linneu. Anais da E.S.A. "Luiz de Queiroz", 31: 677-700.

RUSSEL, P. 1924 - Identification of the commonly cultivated species of Cucurbita by means seed characters. Jour. Wash. Acad. Sci. 14: 265-269.

TEIXEIRA, E.F. 1949 - Normas para o estudo pomológico das frutás mais cultivadas no Brasil. Ministério da Agricultura. $68 \mathrm{p}$.

WHITAKER, T.W.; DAVIS, G.N. 1962 - Cucurbits. London, Leonard Hill, 250 p. 
